\title{
A LAPAROSCOPIC COMPARATIVE STUDY OF RIGHT AND LEFT PARADUODENAL HERNIAE : A TALE OF TWO CITIES
}

\section{General Surgery}

\section{Dr Bhavesh Shelke*}

\section{Dr Abhijit Joshi}

Resident in Dept. of General \& Laparoscopic Surgery, DR L H Hiranandani Hospital, Hillside avenue, Hiranandani Gardens, Powai, Mumbai-400076, India *Corresponding Author

MBBS, MS (Gen Surgery), Fellowship in Laparoscopy, Diploma in Advanced Laparoscopy, Consultant General, G I \& Endo-Laparoscopic Surgeon, DR L H Hiranandani Hospital, Hillside avenue, Hiranandani Gardens, Powai, Mumbai-400076, India

\section{ABSTRACT}

'It was the best of times, it was the worst of times...', thus began Charles Dickens in his classic novel 'A Tale Of Two Cities'. We revisit right and left Paraduodenal herniae(PDH) in this article. Our 'two cities' are the fossae of Waldeyer and Landzert respectively. We think the times are the best as of present day, since cutting edge diagnostic tools(CECT abdomen) offer us an unambiguous diagnosis of PDH. Also the advent of minimal access surgery from the last decade of the twentieth century onwards, has afforded us the luxury of treating PDH by laparoscopy. However these are also the worst of times since PDH, though rare, constitutes $53 \%$ of all internal hernias and if undiagnosed, can increase mortality rate upto $20 \%$ [1]. In this article, we attempt to make a side by side comparison of right and left PDH vis-à-vis their embryology, diagnosis, laparoscopic 'first look appearance', surgical therapy and the endresult.

\section{KEYWORDS}

Right \& left para-duodenal Hernia (PDH), Intestinal obstruction, post- partum, mid-gut rotation.

\section{INTRODUCTION:}

Intestinal obstruction due to internal hernia is very rare. Among internal hernias PDH is very common[1]. Traditionally it has been treated with open surgery, but since the advent of minimal access surgery, laparoscopy is an additional valuable therapeutic tool.

CASE 1: A 29-year-old female with no comorbidities presented with acute abdominal pain radiating to the back, nausea, bilious vomiting, abdominal distention and obstipation since 2 days. She had undergone full term normal delivery four days back. She did not give any history of per rectal bleed. She was stable hemodynamically. Per abdominal examination revealed mild abdominal distension with slight tenderness in the upper abdomen and hyperperistalsis in the central abdomen. Perrectal examination revealed an empty rectum. The laboratory reports were unremarkable, except for leukocytosis [15230 per high power field]. Radiological examination: A] Erect abdominal X ray revealed multiple air fluid levels. B] Ultrasonography [USG] of the abdomen showed dilated small bowel loops and minimal inter-bowel free fluid. $\mathrm{C}]$ Contrast enhanced abdominal computed tomography [CECT] diagnosed a right $\mathrm{PDH}$. The same was repaired laparoscopically.

CASE 2: A 33 years old male patient presented to the casualty ward with colicky pain in left upper abdomen on and off since 15 days, one episode of bilious vomiting and abdominal distension with constipation since 2 days. On examination, his vitals were stable. On per abdominal examination, he had a distended abdomen, tender left hypochondrium and hyperperistalsis in the central abdomen. A per rectal examination revealed an empty rectum. Laboratory reports were unremarkable. X ray abdomen done in standing position showed distended small bowel loops and few air-fluid levels in the left upper abdomen. Ultrasonography of abdomen revealed vague probe tenderness, matted appearance of small bowel loops, pseudokidney appearance and increased omental fat echogenicity. A CECT scan of the abdomen and pelvis diagnosed a left $\mathrm{PDH}$. The same was repaired laparoscopically.

\section{DISCUSSION:}

Internal hernias are abnormal protrusions of part or whole of viscera through defects in the mesentery or peritoneum contained within abdominal cavity [1]. They constitute less than $2 \%$ of all cases of intestinal obstruction [5]. Neubauer was the first to describe Para duodenal hernia in 1786[6]. Para-duodenal hernia is defined as abnormal protrusion of part or whole of viscera into para-duodenal fossa. PDH constitutes less than $1 \%$ of all cases of small intestinal obstruction [1]. 53\% of all internal hernias are PDH [1]. Of all cases of $\mathrm{PDH}$, left PDH constitutes $75 \%$ and right PDH constitutes 25\% [1]. The most common presentation of PDH is in the 4th-5th decade of life, with male to female ratio being $3: 1$ [8]. Patients may present with nonspecific symptoms such as nausea, vomiting, or dull aching long term abdominal pain. However, it may also present with features of bowel obstruction such as vomiting, abdominal distention, sluggish bowel movements, constipation or obstipation. PDH can also present with lump in abdomen. PDH can also be an incidental discovery during operation or radiological imaging [7]. PDH can be treated by open or laparoscopic surgery. The basic principles of the surgery are: 1] Reduction of the hernial contents with or without sac excision, 2] Suture-closure of the defect with or without meshplasty or 3] Marsupialisation, if the size of the defect is large. In our cases, the defects were laid open and the sacs were marsupialised. Topographic anatomical knowledge of the hernial borders is of clinical significance, as one can damage major vessels [SMA in right PDH or IMV in left $\mathrm{PDH}]$ during the repair of the hernia. However, it has been noted that in a case of left PDH, in order to get an access into the hernial orifice one may sacrifice the inferior mesenteric vein [IMV][9]. We were able to manage our cases totally laparoscopically. Laparoscopy has the wellknown advantage of magnified view, less morbidity, shorter hospital stay and early recovery [4]

\section{Comparison of Embryology}

Case 1 - Embryological development of right para-duodenal hernia consists of non-rotation of pre-arterial segment of midgut after initial 90 degree counterclockwise rotation with continued rotation of postarterial segment of midgut which leads to entrapment of small bowel[pre-arterial segment] behind large bowel[post-arterial segment] mesentery in a recess called Waldeyer's fossa( our 'first city'), which lies posterior to ileo-colic, right colic, middle colic vessels and anterior to posterior parietal peritoneum [7].

Case 2 - Left para-duodenal hernia generally affects the male population. It's development consists of normal rotation of gut and abnormal fixation of the descending mesocolon to the posterior abdominal wall, which leads to formation of an unsupported area and herniation of contents into Landzert's fossa(our 'second city'), which lies lateral to the fourth part of the duodenum, posterior to the inferior mesenteric vein [IMV], the ascending branch of the ascending left colic artery [LCA] and anterior to posterior parietal peritoneum [7](Fig 1)
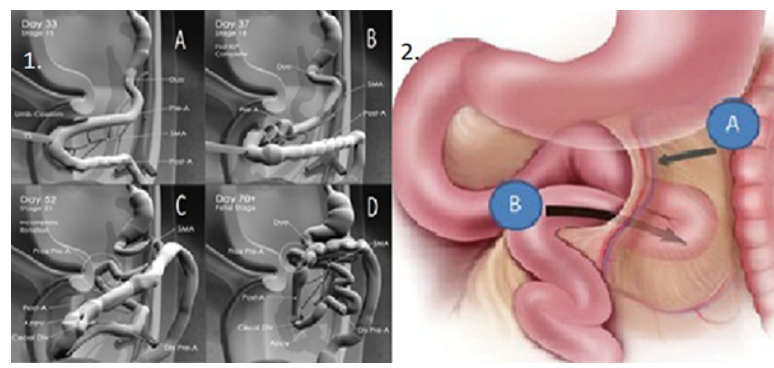

Fig 1 : Comparison Of Embrology- 
Section1(left half of pic): Embryology of right PDH A] Herniation of midgut B] Initial 90 degree rotation of midgut C] Non-rotation of prearterial segment with continued rotation of post-arterial segment D] Entrapment of Pre-arterial segment behind Post-arterial segment

Section 2(right half of pic): Embryology of left PDH: Normal rotation of gut but abnormal fixation of the descending mesocolon to the posterior abdominal wall, which leads to formation of an unsupported area[Landzert's fossa](A) and herniation of contents into it(B).

\section{Comparison of diagnostics:}

The gold standard preoperative diagnostic investigation for PDH is contrast enhanced computed tomography of the abdomen [CECT]

Case 1 - In right $\mathrm{PDH}$, the CECT abdomen identified clustered contrast filled small bowel loops along the right side of the abdomen with features of non-rotation of midgut. SMA, Caecum and ascending mesocolon were anterior to the clustered small bowel. Sometimes SMA, ileocolic artery and right colic vein lie in the anterior margin of the neck of the sac [3]

Case 2 - CECT of left PDH revealed clustered contrast filled small bowel loops seen between stomach or transverse colon anteriorly and pancreas or left adrenal gland posteriorly. Inferior mesenteric vein and ascending branch of left colic artery lie in the anterior or medial border of the sac [3](Fig 2).

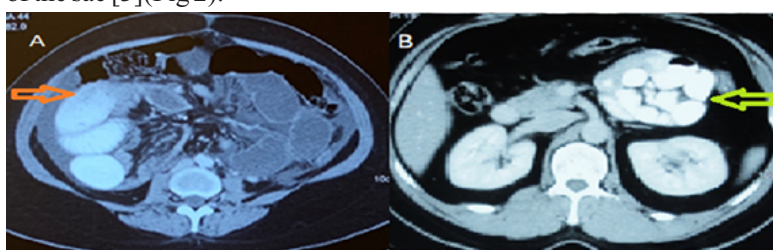

Fig.2 Comparison Of Diagnostic CT Cuts -

A]Right PDH - CECT Abdomen [axial view] showing contrast filled distended small bowel loops behind ascending colon

B]Left PDH - CECT Abdomen [axial view] showing contrast filled clumped small bowel loops behind descending colon

\section{Comparison of therapy:}

Case 1- . At laparoscopy, a long stretch of small bowel loops was seen going into a recess present posterior to ascending colon. These entrapped loops could not be reduced by gentle taxis. Hence lateral attachments of ascending colon were divided. Waldeyer's fossa(our 'first city') was then laid open and marsupialised with the general peritoneal cavity by incising the sac, laterally, in a progressively cephalad direction. Contents of the hernial sac were reduced. The entrapped small bowel was not devitalized. After peritoneal toilet, ample quantity of normal saline was instilled into the peritoneal cavity to reduce the chances of post-operative adhesions. Post-operative recovery was uneventful. Over the ensuing six months, she continues to be symptom free(Fig.3A,B/Fig.4A,B/Fig.5A).

Case 2- At laparoscopy, gentle attempts at reduction yielded favourable results and most of the entrapped small bowel could be reduced back into the generalised peritoneal cavity. The anterior border of the narrow inlet to the fossa which contained the inferior mesenteric vein [IMV] was then divided using ultrasonic shears. This wide division of the neck of the sac thus marsupialised the recess i.e. the fossa of Landzert(our 'second city') and merged it with the peritoneal cavity. The post-operative recovery of the patient was uneventful and he remains asymptomatic over the ensuing five years(Fig.3C,D/Fig.4C,D/Fig.5B)

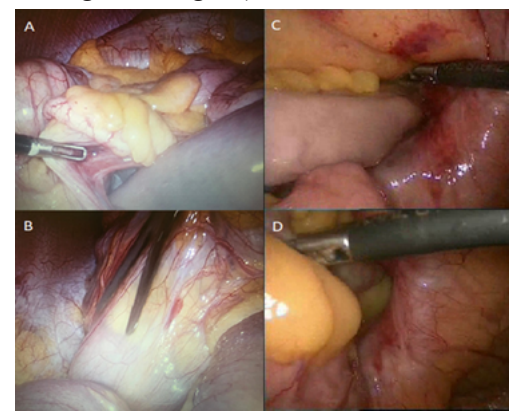

Fig 3 Comparison Of 1st Look Laparoscopy Pics
A\&B]Rt PDH-A] Small bowel loops seen going into Waldeyer's fossa B] Attempted but failed reduction of hernial contents by gentle taxis

C\&D]Lt PDH-C] Small bowel loops entering into the Landzert's fossa D] Small bowel loops being delivered out of Landzert's fossa.

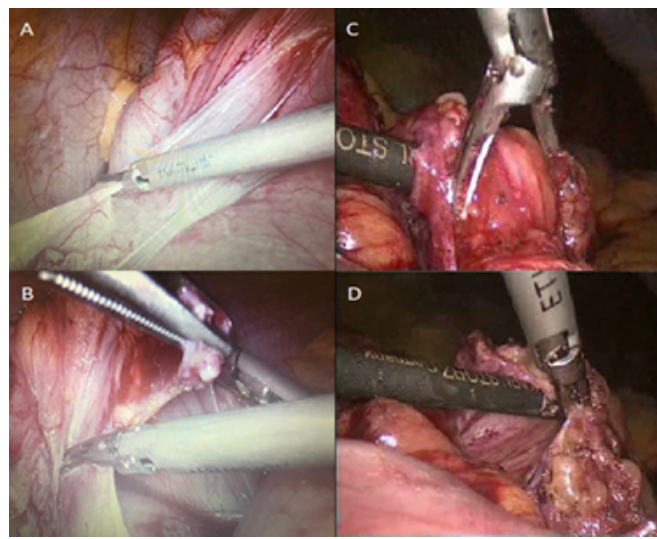

Fig 4 Comparison Of Laparoscopic Therapy

A\&B] For right PDH: A] Division of lateral attachments of ascending colon and marsupialisation of sac B] Whole hernia sac is merged with the peritoneal cavity

C\&D] For left PDH: C] Anterior border of inlet to Landzert's fossa being lifted up \& presented to the ultrasonic shears D] Anterior border of inlet to Landzert's fossa being divided

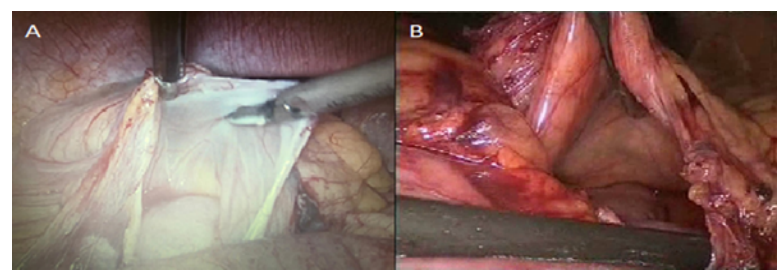

Fig.5 Comparison Of The Endresults

A]Right PDH: Marsupialised fossa of Waldeyer (our'1st city')

B]Left PDH: Marsupialised fossa of Landzert (our '2nd city')

\section{CONCLUSION:}

In a case of intestinal obstruction; $\mathrm{PDH}$, though a rare entity, should be considered as a differential diagnosis. However, rarity and nonspecific symptoms are the two factors which present a difficulty in clinical diagnosis of PDH. A CECT abdomen is the diagnostic clincher in PDH and has a high specificity for diagnosis. Even in emergency presentations, $\mathrm{PDH}$ can be managed successfully with laparoscopy.

\section{List of abbreviations:}

CECT: Contrast Enhanced Computerised Tomography Scan PDH: Para-duodenal Hernia

AP: Antero-Posterior

USG: Ultrasonography

Fig: Figure

NBM: Nil By Mouth

IV: Intravenous

$\mathrm{Cm}$ : Centimeters

$\mathrm{mm}$ : Milimeters

PDH: Paraduodenal Hernia

SMA: Superior Mesenteric Artery

SMV: Superior Mesenteric Vein

LCA: Left Colic Artery

IMV: Inferior Mesenteric Vein

Consent for Publication: Written Informed consent has been taken from patients for publication for their cases in medical journal.

Ethical Approval: Ethical approval is not required at our institution for such case-report publication where identity is not revealed.

\section{REFERENCES:}

Rahaf S, Huda S, Yazeed B, Haifaa M. Rare Case of Small Bowel Obstruction Due to Paraduodenal Hernia Am J Case Rep. 2019; 20: 1581-1586 doi: 10.12659/ AJCR. 918403

. Patil R, Smith C, Brown MD. Para-duodenal hernia presenting as unexplained recurren International Journal of Scientific Research 
abdominal pain. Am J Gastroenterology 1999; 94:3614-5

3. Martin LC, Merkle EM, Thompson WM. Review of internal hernias: radiographic and clinical findings. AJR Am J Roentgenol. 2006;186 (3): 703-17. doi:10.2214/ AJR. 05.0644 - Pubmed citation

4. Shadhu K, Ramlagun D \& Ping, X. Para-duodenal hernia: a report of five cases and review of literature. BMC Surg 18, 32 2[018]. https://doi.org/10.1186/s12893-0180365-8

5. S. Akbulut, "Unusual cause of intestinal obstruction: left paraduodenal hernia," Case Reports in Medicine, vol. 2012, Article ID 529246, 2 pages, 2012

Rohit M, Aswini K Right paraduodenal hernia: report of two cases and review of literature http://www.journalofmas.com/text.asp?2010/6/4/122/72601

7. Bartlett MK, Wang C, Williams WH. The surgical management of paraduodenal hernia. Ann Surg 1968; 168:249-54.

8. Mathew A, Oliver M. Left Paraduodenal Hernia: A Rare Complication following Laparoscopic Appendectomy DOI: 10.1155/2017/3913784

9. Parmar B, Parmar R. Laparoscopic management of left paraduodenal hernia. J Min Access Surg [serial online] 2010 [cited 2020 May 21]; 6:122-4SS 